Series A

I. MATHEMATICA

$336 / 8$

CLUSTER SET THEOREMS FOR ARBITRARY FUNCTIONS WITH APPLICATIONS TO FUNCTION THEORY

BY

E. F. COLLINGWOOD

H E L S I K I 1963

S U O M A L A I N E N T I E DEA K A D E M I A 
Communicated 11 January 1963 by P. J. Myrberg and Olli Lehto 


\section{Cluster set theorems for arbitrary functions with applications to function theory}

1. Introduction. At first sight a function of which we know no more than that it has a value at every point of its domain looks rather unpromising material to work with. There are obvious limitations; but it has been found that quite striking statements can be made about such functions and if we select them to fit situations that arise, for example, in the theory of functions these statements may turn out to be useful theorems. Or it may be that a theorem about an arbitrary function can be significantly sharpened if some condition such as continuity is imposed. In any case "arbitrariness» is qualified by the properties of the spaces one of which is mapped into the other by the function. Here we shall for the most part be concerned with mappings from the real line or the plane disc to the 2-sphere or a great circle upon it, thus generalising a familiar function - theoretic situation. The theorems we obtain are inherent in the topology and geometry of the spaces involved and, as J. D. Weston has shown [18], are capable of further generalisation by removing restrictions on these spaces. Important as these developments are we shall not attempt to enter into them here.

2. The theorems we shall discuss are in terms of cluster sets; but these are not the only kind of theorems about arbitrary functions that are known. We denote the functions by $f(z),|z|<1 ; f(x), 0<x<1 ; f(P)$ or simply $f$. The cluster set of $f$ at a point $P$ of the closure of its domain, which we denote by $C(f, P)$, is the set of all the limits of $f$ as the variable $z$ or $x$ within the domain $D$ of $f$ approaches $P$ in all possible ways. In precise terms, $C(f, P), P \in \bar{D}$, may be defined in either of the two following equivalent ways:

(a) $C(f, P)$ is the set of points $\alpha$ of the $w$-sphere $S$ into which $w=f(z) \quad$ or $\quad w=f(x)$ maps $D$ such that there exists a sequence $\left\{z_{n}\right\} \subset D \backslash P$ satisfying $\lim _{n \rightarrow \infty} z_{n}=P$ and $\lim _{n \rightarrow \infty} f\left(z_{n}\right)=\alpha$.

(b) $C(f, P)=\cap \bar{\Delta}_{n}$ where $\Delta_{n}=f\left(\mathscr{D}_{n} \backslash P\right)$ and $\mathscr{D}_{n}$ is the intersection of $D$ with a neighbourhood $|x-P|<\delta_{n}$, or $|z-P|<\delta_{n}, \quad \delta_{n} \downarrow 0$ as $n \rightarrow \infty$. The open sets $\mathscr{D}_{n}, n=1,2, \ldots$, thus have $P$ as their only common interior or frontier point. The bar denotes closure. 
It follows at once from both definitions that $C(f, P)$ is non-empty and closed. The complement $\mathcal{C} C(f, P)$ is thus always an open set.

We note that if $f(z)$ is defined at $P$ then $f(P)$ does not necessarily belong to $C(f, P)$ unless $f$ is continuous at $P$. But if $f(z)$ is continuous in the deleted set $D \backslash P$ then $C(f, P)$ is either a continuum (compact connected set) or a point. In the latter case we say that $C(f, P)$ is degenerate; and in the former we say that $C(f, P)$ is total or sub-total according as $\mathcal{C} C(f, P)$ is empty or not.

The cluster set as we have defined it is complete, being relative to the entire neighbourhood of $P$ belonging to the domain $D$. But restricted cluster sets (these are sometimes called partial) are important in the theory. Examples are the radial cluster set $C_{\varrho}(f, P)$, where $D$ is a disc and $P$ a point of its circumference, defined by restricting $\left\{z_{n}\right\}$ to the radius to $P$, and the right and left-hand cluster sets $C_{R}(f, P)$ and $C_{L}(f, P)$, where $x_{n}$ approaches $P$ from the right (left) for $f$ defined on the real line and $\arg z_{n}$ approaches arg $P$ from below (above) for $f$ defined in a disc and $P$ a point of the circumference.

\section{Symmetry theorems}

3. In 1907 W. H. Young [19] proved the following theorem.

If $f(x)$ is an arbitrary real function of the real variable $x$ then

$$
\varlimsup f_{L}(x)=\varlimsup \lim f_{R}(x) \quad \text { and } \quad \underline{\lim } f_{L}(x)=\underline{\lim } f_{R}(x),
$$

where the $R$ and $L$ signs denote limits to the right and left of $x$ respectively, for all values of $x$ except perhaps for a countable set.

This appears to have been the first theorem to be explicitly stated for arbitrary functions. Young announced it at the meeting of the British Association held at Leicester in 1907. He used to refer to it as the Leicester theorem. A year later, at the Rome Congress of 1908, he announced what he called the Rome theorem [20]. Stated in modern notation, this is the Leicester theorem with (1) replaced by

$$
C_{L}(f, x)=C_{R}(f, x) \text {. }
$$

Young extended his investigations to the plane and to euclidean $n$-space in a series of papers [19]- [24] extending over twenty years; but he confined himself to real functions and his results passed unnoticed by complex variable analysts (see also [16]).

4. What turns out to be a useful extension of Young's Rome theorem to an arbitrary complex function defined in the disc is, however, almost 
immediate. Denote the unit disc by $U$ and its boundary by $\varkappa$. Writing $z_{n}=r_{n} e^{i \vartheta_{n}}$ the right (left) cluster set of $f(z)$ at $P \in \varkappa$ is defined as the set of values $\alpha$ such that for some sequence $\left\{z_{n}\right\} \subset U, \lim z_{n}=P$, $\vartheta_{n} \uparrow \arg P \quad\left(\vartheta_{n} \downarrow \arg P\right)$, we have $\lim _{n \rightarrow \infty} f\left(z_{n}\right)=\alpha$. We can now prove

Theorem 1. For an arbitrary real or complex function $f(z)$ defined in $U$

$$
C_{R}\left(f, e^{i \vartheta}\right)=C_{L}\left(f, e^{i \vartheta}\right)=C\left(f, e^{i \vartheta}\right)
$$

except perhaps for a countable set of points $e^{i \vartheta} \in \varkappa$.

The proof, which also suffices, without any adaptation, to prove Young's Rome theorem, is simple.

We cover the Riemann sphere $S$ with a succession of nets $N_{k}, \quad k=1,2, \ldots$, each with a finite number of closed triangular meshes $m_{k n}, \quad n=1,2, \ldots, n_{k}$, of diameter $<1 / \mathrm{k}$. We arrange these in a sequence in any convenient way. Call the sequence $\left\{m_{j}\right\}$.

For each index $j$ we define the set $E_{j}$ of points $e^{i \vartheta}$ for which $m_{j}$ meets $C\left(f, e^{i \vartheta}\right)$ but does not meet $C_{R}\left(f, e^{i \vartheta}\right)$. If $e^{i \vartheta} \in E_{j}$, then $e^{i \vartheta}$ is the left-hand end-point of an $\operatorname{arc} I_{j}(\vartheta)$ such that for $e^{i \psi} \in I_{j}(\vartheta)$ the cluster set $C\left(f, e^{i \psi^{\prime}}\right)$ does not meet $m_{j}$; for otherwise $m_{j}$ would meet $C_{R}\left(f, e^{i \vartheta}\right)$. $I_{j}(\vartheta)$ therefore contains no point of $E_{j}$. The set $E_{j}$ thus consists of end-points of non-overlapping intervals and is therefore countable. Plainly, the set $E_{R}$ of points $e^{i \vartheta}$ for which $C\left(f, e^{i \vartheta}\right) \backslash C_{R}\left(f, e^{i \vartheta}\right)$ is not empty is the union of the sets $E_{j}, j=1,2, \ldots$, and this proves the theorem.

5. Exactly the same argument proves the local symmetry, except perhaps at a countable set, of the right and left boundary cluster sets. The right hand boundary cluster set $C_{B R}\left(f, e^{i \vartheta}\right)$ at $e^{i \vartheta}$ is simply the cluster set defined along the arc of $x$ to the right of $e^{i v}$, only, since $f$ is not necessarily defined at points of $x$, we use the cluster sets of $f$ at points of the are as the values. Any appearance of sophistication in this definition is deceptive. Similarly for the left hand boundary cluster set $C_{B L}\left(f, e^{i \vartheta}\right)$. We then have

Theorem 2. For an arbitrary function $f(z)$ in $U$

$$
C_{B R}\left(f, e^{i \vartheta}\right)=C_{B L}\left(f, e^{i \vartheta}\right)=C\left(f, e^{i \vartheta}\right)
$$

except perhaps for a countable set of points $e^{i \vartheta} \in \varkappa$.

Since evidently $C_{B L}\left(f, e^{i \vartheta}\right) \subset C_{L}\left(f, e^{i \vartheta}\right)$ and $C_{B R}\left(f, e^{i \vartheta}\right) \subset C_{R}\left(f, e^{i \vartheta}\right)$ Theorem 1 is contained in Theorem 2 .

It will be seen that Theorems 1 and 2 and Young's theorems quoted above depend essentially on the topology of the real line or circumference of the disc. However, for functions defined in a ball we can, by the same 
argument, establish bilateral symmetry of the global cluster sets and global boundary cluster sets to the right and left of a meridian, say, or parallel of latitude (looking respectively eastwards or northwards, say) except for a countable set of values of the longitude or latitude. By the global cluster set to the right of a meridian we mean the union of the cluster sets at all points of the meridian approached from the right, and similarly for the other one-sided global cluster sets. Patterns of symmetry at points of the spherical boundary of the ball, equality in opposite quadrants defined by the meridian and parallel through the point, for example, may be shown by an obvious adaptation of Young's method for the plane [23] to hold except for a set of points of first category on a countable set of meridians or parallels. In contrast, Bagemihl's ambiguous point theorem for an arbitrary function in the disc is essentially 2-dimensional and has no analogue in the ball.

\section{Maximality theorems}

6. It follows from (4) that the cluster sets at $e^{i \text { i }}$ in the two domains in $U$ lying outside chords intersecting at $e^{i \vartheta}$ and to the right and left of the radius are both maximal, i. e. equal to $C\left(f, e^{i \vartheta}\right)$, except for a countable set of values of $\vartheta$. This suggests the question: What can we say about the cluster set in a small angle $\Delta$ at $e^{i \vartheta}$ between two chords? To fix ideas suppose that $\Delta$ is bisected by the radius $\varrho$. In this situation the kind of argument by which we proved Theorem 1 is not available and the set of points $e^{i \vartheta} \in \varkappa$ at which $C_{\Delta}\left(f, e^{i \vartheta}\right) \neq C\left(f, e^{i \vartheta}\right)$ is no longer countable but is of first category (we shall write this category I) in the sense of Baire. The theorem [10] is as follows:

Theorem 3. For an arbitrary function $f(z)$ in $U^{+}$and an angle $\Delta(\vartheta)$ of any fixed magnitude bisected by the radius to $e^{i, 7}$

$$
C_{\Delta(\vartheta)}\left(f, e^{i \vartheta}\right)=C\left(f, e^{i / f}\right)
$$

except perhaps for a set of points of category $I$ on $\%$.

Suppose the contrary so that the set $E$ of points $e^{i y^{4}}$ at which $C_{\Delta(\vartheta)}\left(f, e^{i \vartheta}\right) \neq C\left(f, e^{i \vartheta}\right)$ is of category II. In three steps we shall select a subset $E_{0}$ of $E$, also of category II and therefore dense in an arc $\beta$ of $x$, in which a certain closed subset $T_{0}$ of the 2 -sphere $S$ meets $C\left(f, e^{i \vartheta}\right)$ but is uniformly bounded away from $f(\Delta(\vartheta))$. These properties are inconsistent. For, since $E_{0}$ is dense in $\beta$ every point $z$ of $U$ sufficieutly near to $\beta$ is contained in a $\Delta(\vartheta), e^{i \eta} \in E_{0}$, so that $f(z)$ is uniformly bounded away from $T_{0}$ which therefore does not meet $C\left(f, e^{i i^{i}}\right)$ for any $e^{i \vartheta} \in \beta$. This contradiction will prove the theorem. 
The selection is carried out as follows.

(i) For any point of $E$, since $C_{\Delta(\vartheta)}\left(f, e^{i \vartheta}\right)$ is closed and $\subset C\left(f, e^{i \vartheta}\right)$, there is a positive number $\varepsilon$ such that the intersection

$$
C\left(f, e^{i \vartheta}\right) \cap \mathcal{C}\left(C_{\Delta(\vartheta)}\left(f, e^{i \vartheta}\right)_{+\varepsilon}\right)
$$

is not empty, where $C_{A(\vartheta)}\left(f, e^{i \vartheta}\right)_{+\varepsilon}$ is the closed $\varepsilon$-neighbourhood of $C_{\Delta(\vartheta)}\left(f, e^{i, \vartheta}\right)$ (in the spherical metric) and $\odot$ denotes the complement. Choose a sequence $\varepsilon_{1}>\varepsilon_{2}>\ldots>\varepsilon_{n}>\ldots, \lim \varepsilon_{n}=0$, and denote by $E_{n}$ the subset of $E$ for which

$$
C\left(f, e^{i \vartheta}\right) \cap \mathcal{C}\left(C_{\Delta(\vartheta)}\left(f, e^{i \vartheta}\right)_{+\varepsilon_{n}}\right)
$$

is not empty. Plainly, $E_{1} \subset E_{2} \subset \ldots \subset E_{n} \subset \ldots$ (a finite number of these sets may be empty) and

$$
E=\bigcup_{n} E_{n}
$$

We can therefore find $N$ such that $E_{N}$ (and every set of higher rank) is of category II.

(ii) To select from $E_{N}$ we now cover $S$ with a finite net of meshes $T_{1}, T_{2}, \ldots, T_{m}$ each of diameter less than $\varepsilon_{N} / 4$. We treat the meshes $T$ as closed. For a given $\mu \leqq m$ let $E_{N_{\mu}}$ be the subset of $E_{N}$ in which the set

$$
T_{\mu} \cap C\left(f, e^{i \vartheta}\right) \cap \mathcal{C}\left(C_{\Delta(\vartheta)}\left(f, e^{i \vartheta}\right)_{+\varepsilon_{N}}\right)
$$

is not empty $\left(T_{\mu}\right.$ meets $C\left(f, e^{i \vartheta}\right)$ but not $\left.C_{\Delta(\vartheta \vartheta}\left(f, e^{i \vartheta}\right)_{+\varepsilon_{N}}\right)$. Since

$$
E_{N}=\bigcup_{u \leqq m} E_{N \mu}
$$

and $E_{N}$ is of category II we can find $M \leqq m$ such that $E_{N M}$ is of category II.

(iii) Finally we have to select from $E_{N M}$. Let $T_{0}$ be any closed subset of $T_{M}$. Then for all $e^{i,} \in E_{N M}$ the distance of $T_{0}$ from $C_{\Delta(\vartheta)}\left(f, e^{i \vartheta}\right)$ is greater than $3 \varepsilon_{N} / 4$. We define $E_{N M q}, q=1,2, \ldots$, as the subset of $E_{N M}$ in which the (spherical) distance $\left[f(z), T_{0}\right]$ of $f(z)$ from $T_{0}$ satisfies the inequality $\left[f(z), T_{0}\right]>\varepsilon_{N} / 2$ for all $z \in \Delta(\vartheta), 1-2^{-q}<|z|<1$. Again,

$$
E_{\mathrm{NM} 1} \subset E_{\mathrm{NM} 2} \subset \ldots \subset E_{N M q} \subset \ldots
$$

and

$$
E_{N M}=\underset{q}{\cup} E_{N M q}
$$

Since $E_{N M}$ is of category II we can find $Q$ such that $E_{N M Q}$ is of category II and therefore dense in some arc $\beta \subset \varkappa$. To complete the proof of the theorem we have only to put $E_{0}=E_{N M Q}$. 
7. The argument by which we have proved Theorem 3 may be applied to any angle of fixed magnitude and orientation relative to the radius. We can specify a countable set of directions and magnitudes so that any angle with $e^{i \vartheta}$ as vertex contains one of the angles so specified. We therefore have

Theorem 4. Let $f(z)$ be an arbitrary function in $U$. Then except perhaps for a set $E$ of points $e^{i,}$ of category $I$ on $\%$ we have for every angle $\Delta \subset U$ with $e^{i,}$ as vertex

$$
C_{\Delta}\left(f, e^{i \vartheta}\right)=C\left(f, e^{i \vartheta}\right) .
$$

At a point $e^{i \vartheta} \in \mathcal{X} \backslash E$ at which $C\left(f, e^{i \vartheta}\right)$ is total $f(z)$ has the cluster set property which a meromorphic function has at a Plessner point [9]. It must be emphasised, however, that Theorem 4 is in no way comparable with Plessner's well known theorem for meromorphic functions.

8. If we now impose the condition of continuity on $f(z)$ a quite trivial change in the proof of Theorem 3, working with $C_{g}\left(f, e^{i \vartheta}\right)$ instead of $C_{\Delta(\vartheta)}\left(f, e^{i \vartheta}\right)$ gives the following maximality theorem [9]

Theorem 5. If $f(z)$ is continuous in $U$ then

$$
C_{\varrho}\left(f, e^{i \vartheta}\right)=C\left(f, e^{i \vartheta}\right)
$$

except perhaps for a set of points of category $I$ on $\varkappa$.

By the same process of selection as in the proof of Theorem 3 we obtain a set $E_{0}$ dense in an arc $\beta \subset \varkappa$ in which $T_{0}$ meets $C\left(f, e^{i \vartheta}\right)$ but is uniformly bounded away from $f\left(r e^{i \vartheta}\right), e^{i \vartheta} \in E_{0}, 1-2^{-Q}<r<1$. By the continuity of $f(z)$ these properties are inconsistent and the theorem is proved.

Theorems 3 and 5 are special cases of more general results proved by essentially the same arguments $[9]-[10]$.

\section{Applications}

9. An obvious application of Theorem 5 was to Caratheodory's problem of determining the distribution of the four kinds of prime ends which correspond under a conformal mapping of $U$ onto a simply connected domain, not necessarily Jordan, to points of $\%$. The correspondence is $1: 1$ and the impression of the prime end corresponding to a point $e^{i \vartheta} \in \varkappa$ under a conformal mapping $f(z)$ is the cluster set $C\left(f, e^{i \vartheta}\right)$. If $\lambda$ is any path in $U$ terminating at $e^{i \vartheta}$ then plainly $C_{j}\left(f, e^{i \vartheta}\right) \subseteq C\left(f, e^{i \vartheta}\right)$. A point of $C\left(f, e^{i \vartheta}\right)$ which belongs to every cluster set $C_{i}\left(f, e^{i \vartheta}\right)$ is a principal point of the prime end in question. Points of $C\left(f, e^{i \vartheta}\right)$ which are not 
principal are subsidiary. The set $\Pi\left(f, e^{i \vartheta}\right)$ of principal points of the prime end is either degenerate, in which case the point is accessible from inside $U$ and is the sole accessible point of the prime end, or is a continuum, in which case there are no accessible points of the prime end.

A prime end is of the first kind if it consists of a single (necessarily) accessible point so that $C\left(f, e^{i \vartheta}\right)$ is degenerate. It is of the second kind if it has one (accessible) principal point and an infinity of subsidiary points; of the third kind if it has no subsidiary points and an infinity of principal points; and of the fourth kind if it has an infinity of both principal and subsidiary points. We denote these four classes of prime ends by $\mathscr{O}_{1}, \mathscr{O}_{2}, \mathscr{O}_{3}, \mathscr{O}_{4}$, and the corresponding sets on $x$ by $e_{1}, e_{2}, e_{3}, e_{4}$.

It was proved by Lindelöf that for any conformal mapping $f(z)$ and any point $e^{i \vartheta}$

$$
C_{\varrho}\left(f, e^{i \vartheta}\right)=\Pi\left(f, e^{i \vartheta}\right) .
$$

Combining (8) with (9) we see that

$$
\Pi\left(f, e^{i \vartheta}\right)=C\left(f, e^{i \vartheta}\right)
$$

except perhaps for a set of points $e^{i \vartheta}$ of category $I$ on $\varkappa$. The points for which (10) is satisfied are precisely the points $e_{1} \cup e_{3}$ corresponding to $\mathscr{O}_{1} \cup \mathscr{O}_{3}$ so that the complementary sets $e_{2} \cup e_{4}$ in $\varkappa$ and $\mathscr{O}_{2} \cup \mathscr{O}_{4}$ in the metric space of prime ends, the metric being, for example, the chordal distance between corresponding points of $\varkappa$, are both of category I. This result is one of the keys to the solution of the distribution problem the implications of which have been described elsewhere [9] and need not be repeated here.

10. A recent refinement of the distribution problem concerns the relative frequency of asymmetrical prime ends. There can be no asymmetry about a prime end of the first or third kind. But a prime end of the second or fourth kind is asymmetrical if its right and left wings are not identical, which is equivalent to saying that $C_{R}\left(f, e^{i \vartheta}\right) \neq C_{L}\left(f, e^{i \vartheta}\right)$. This question is solved by Theorem 1, which is so fundamental as to be valid for an arbitrary function. It shows that the asymmetrical prime ends of any domain are actually countable; and examples show that they may be infinite in number and their corresponding points dense on $x$ [11].

11. Unlike the symmetry theorems 1 and 2 the maximality theorems 3 , 4 and 5 may be immediately extended to functions defined in a three-dimensional ball, where now $\Delta(P)$ will denote a cone having $P$, a point of the spherical boundary of the ball, as its vertex and the radius to this point as its axis. The 2 -sphere being a complete metric space, the Baire category 
theorem is again applicable and the arguments used to prove Theorem 3, with only the obvious verbal changes, give

Theorem 6. For an arbitrary function $f$ which maps the unit ball into 3-space we have

$$
C_{\Delta(P)}(f, P)=C(f, P)
$$

except perhaps for a set of points $P$ of category $I$ on the spherical boundary $S$ of the ball.

The definitions of the cluster sets featuring in this theorem are obvious.

Similarly, if $f$ is continuous in the ball and $C_{o}(f, P)$ is the radial cluster set at $P$ we have

Theorem 7. If the function $f$ is continuous in the unit ball, then

$$
C_{0}(f, P)=C(f, P)
$$

except perhaps for a set of points $P$ of category $I$ on $S$.

This last result is a special case of a general theorem of Weston's on cluster sets of mappings from one topological space to another. For an abstract theory of cluster sets in a general topological setting we refer to Weston [18].

Theorem 7, being sufficiently fundamental to be valid for any continuous function is applicable, for example, to quasiconformal mappings of the ball in space.

12. Let $D(f)$ denote the set of points of $x$ for which $C_{\varrho}\left(f, e^{i \vartheta}\right)$ is degenerate, i. e. $\lim _{r \rightarrow 1} f\left(r e^{i \vartheta}\right)$ exists. We denote this limit by $f\left(e^{i \vartheta}\right)$; and following Doob [12] we may call $\mathscr{F}\left(e^{i \vartheta}\right)=C\left(f, e^{i \vartheta}\right)$ the cluster boundary function for $f$. The following theorem is due to Weniaminoff [17].

If $f(z)$ is analytic and bounded in $U$ and if $* \subset D(f)$, then $C\left(f, e^{i \vartheta}\right)$ is degenerate at every point of $*$ at which $f\left(e^{i f}\right)$ is continuous and hence $e^{i \vartheta} \quad$ is a point of continuity of $\mathscr{J}\left(e^{i \vartheta}\right)$.

Weniaminoff also proves (his Lemma 1 in [17]) that if $x \subset D(f)$ then for an analytic function (evidently in fact for a continuous function) $f\left(e^{i \vartheta}\right)$ is of Baire class 1 and so, by a theorem of Baire, its discontinuities are a set of category I. From this it follows, by the theorem of Weniaminoff quoted above, that

If $f(z)$ is analytic and bounded in $U$ and if $* \subset D(f)$, then $f\left(e^{i, 9}\right)$ being of Baire class 1 is pointwise discontinuous on $\%$, having a residual set of points of continuity on $x$ which are also points of continuity of $\bar{F}\left(e^{i \vartheta}\right)$.

Theorem 5 enables us to generalise this theorem by dropping the requirements that $f(z)$ be bounded or analytic. It is enough that $f(z)$ should be continuous. 
Suppose then that $f(z)$ is continuous in $U$ and that $x \subset D(f)$. Then, by Theorem $5, C\left(f, e^{i \vartheta}\right)$ is degenerate on a residual set i. e. the complement of a set of eategory I on $\varkappa$. But a point at which $C\left(f, e^{i \vartheta}\right)$ is degenerate is a point of continuity of both $\mathscr{F}\left(e^{i \vartheta}\right)$ and $f\left(e^{i \vartheta}\right)$. Thus both $\mathcal{F}\left(e^{i \vartheta}\right)$ and $f\left(e^{i \vartheta}\right)$ are pointwise discontinuous and the set of discontinuities is in each case of category I. By a well known theorem the set of points of continuity of a single valued function is a $G_{\delta}$ (Hausdorff [13], p. 251). The same argument shows that the points of continuity of $\mathscr{F}\left(e^{i \hat{\psi})}\right)$ and $f\left(e^{i \vartheta}\right)$ are both of type $G_{\partial}$. A precisely similar argument applies to a function continuous in the ball and so we have

Theorem 8. If the function $f$ is continuous in the disc (or the ball) and if $x \subset D(f) \quad($ or $S \subset D(f))$ then the cluster boundary function $\mathscr{T}\left(e^{i \vartheta}\right)$ $(\mathscr{S}(P), P \in S)$ is pointwise discontinuous on $\varkappa($ on $S)$ and the set of its discontinuities is an $F_{\sigma}$ of category $I$.

13. We denote by $\Gamma_{\varrho}(f, A)$ the set $f\left(e^{i \vartheta}\right), e^{i \vartheta} \in D(f) \subset A$, of radial limits of $f(z)$ on an are $A \subset x$. The following theorem (proved in [8]), related to Theorem 8 , is also a consequence of Theorem 5 .

Theorem 9. If $f(z)$ is meromorphic in $U$ and $D(f)$ is of category II on some arc $A \subset \varkappa$, then either $\Gamma_{g}(f, A)$ is of positive linear measure or $f(z)=$ constant.

This result is considerably stronger than an earlier theorem of Privalov ([15], pp. 231-232), The conclusion on the measure of $\Gamma_{\rho}(f, A)$ depends upon a lemma of M. L. Cartwright's on meromorphic functions so that the theorem is deeper than the much more general Theorem 8 .

\section{Interior theorems}

14. For the purpose of function-theoretic applications we fix our attention, in considering an arbitrary function, on its properties at the boundary of its domain of definition since it is only there that the regularity of an analytic function breaks down. There are, however, properties of an arbitrary function within its domain which are of independent interest. These are of the kind first brought to light by W. H. Young for real functions and further studied by H. Blumberg [2]-[7]. Variants of them, or some of them, are very easily proved by the methods of this paper for complex functions and for more general mappings.

Let $f(z)$ be an arbitrary function defined in a plane domain $D$ which it maps into the 2 -sphere $S$ (or into the complete euclidean $n$-space) and let $P$ be a point of $D$. We prove 
Theorem 10. If $f(z)$ is an arbitrary function mapping $D$ into $S$ then $f(P) \in C(f, P)$ for all $P$ in $D$ except perhaps for a set which is at most countable.

The proof follows familiar lines. $E$ is now the set of points $P \in D$ in which $f(P) \notin C(f, P)$ so that for all $P \in E$ there is a positive $\varepsilon$ such that the distance $[f(P), C(f, P)]$ of $f(P)$ from $C(f, P)$ (in any appropriate metric) exceeds $\varepsilon$. Given a decreasing sequence $\left\{\varepsilon_{n}\right\}, \lim \varepsilon_{n}=0$, $E_{n}$ is the set such that

$$
[f(P), C(f, P)]>\varepsilon_{n}
$$

so that $E_{1} \subset E_{2} \subset \ldots \subset E_{n} \subset \ldots$ and

$$
E=\cup E_{n}
$$

Let $T_{1}, T_{2}, \ldots, T_{m n}$ be a finite triangulation on $S$ whose meshes are all of diameter less than $\varepsilon_{n} / 4$; and let $E_{n \mu}$ be the subset of $E_{n}$ at every point of which $f(P) \in T_{\mu}, \mu \leqq m n$. Then every $E_{n u}$ is an isolated set. For suppose a point $P_{0}$ of $E_{n \mu}$ to be a limit point of a sequence of points $P_{v} \in E_{n u}$ so that $C\left(f, P_{0}\right)$ meets $T_{\mu}$. Then the distance of $f\left(P_{0}\right)$ from $C\left(f, P_{0}\right)$ is less than $\varepsilon_{n} / 4$, contrary to the definition of $E_{n}$ which contains $E_{n \mu}$. Thus every set $E_{n \mu}$ is countable; hence $E_{n}=\cup E_{n \mu}$ is countable and consequently $E=\cup E_{n}$ is countable, which proves the theorem.

In the case of a function of a real variable $D$ is a segment of the real line and exactly the same argument applies and the exceptional set is again countable. The theorem is also true for an arbitrary mapping into any complete metric space.

This theorem generalises a theorem of Young's (Theorem 1 of [21] and $2^{\circ}$ on page 5 of [22]). It is also easy, by our general method, to prove a rather stronger result of Blumberg's (Theorem 1 of [3]) which he proved for real functions but which is also valid for an arbitrary mapping into $S$ (or into any complete metric space). He introduces the notion of dense approach to a value of the function. The definition is as follows. The function $f$ is said to be densely approached at the point $P$ if for every $\varepsilon>0$ there e rists a neighbourhood $G(P, \varepsilon)$ of $P$ such that the points of this neighbourhood for which

$$
[f(z), f(P)]<\varepsilon
$$

are dense in $G(P, \varepsilon)$. With this definition we have

Theorem 11. If $f(z)$ is an arbitrary function mapping $D$ into $S$ (or any complete metric space) then the set of points at which the value of $f$ is densely approached is residual in $D$.

The set of dense approach is of course a subset of the set for which $f(P) \in C(f, P)$ whose complement is countable. Now let $E$ be the set in 
$D$ at which $f(P)$ is not densely approached. For a given $\varepsilon_{n}$, where again $\left\{\varepsilon_{n}\right\}$ is a decreasing sequence tending to zero, we denote by $E_{n}$ the subset of $E$ such that every neighbourhood of $P \in E_{n}$ contains a domain in which the distance from $f(P)$ to $f(z)$ exceeds $\varepsilon_{n}$. Evidently, $E_{1} \subset E_{2} \subset \ldots \subset E_{n} \subset \ldots$ and $E=\cup E_{n}$. Repeating the triangulations $T_{1}, T_{2}, \ldots, T_{m n}$, the meshes being all of diameter $<\varepsilon_{n} / 4$, the set $E_{n \mu}$ is again the subset of $E_{n}$ in which $f(P) \in T_{\mu}, \mu \leqq m n$. If now the theorem is false the set $E$ of exceptional points is of category II and it follows as before that there exist $n=N$ and $\mu=M$ such that $E_{N M}$ is of category II and therefore dense in a domain $G \subset D$. Let $P_{0}$ be a point of $G \cap E_{N M}$. At every other point of $G \cap E_{N M}$ and at $P_{0}$ itself $f \in T_{M}$ so that the distance of $f\left(P_{0}\right)$ from $f(z), z \in G \cap E_{N M}$, is less than $\varepsilon_{N} / 4$ and so there is no sequence of domains having $P_{0}$ as a limit point in which the distance from $f\left(P_{0}\right)$ to $f(z)$ exceeds $\varepsilon_{N}$ : Since $P_{0} \in E_{N}$ this is a contradiction and the theorem is proved.

\section{Concluding remarks}

15. The earlier literature on arbitrary functions, which is quite considerable, was concerned exclusively with the interior theory. Sufficient references to trace this theory back to its sources are given in the list of references at the end of this paper. The more recent work has been almost as heavily biassed towards boundary theory owing to the discovery, largely through the development of the theory of cluster sets, of applications in the theory of functions and allied fields. The interior theory has, however, also attracted attention as has the extension of the theory to general topological spaces. The lack of a unifying idea, namely that of the cluster set, or of an accepted terminology and notation hampered development for a long time. As has been shown here the descriptive theory may be handled very easily by a uniform method which is applicable to very general situations. The part of the theory which we have not touched on is the metrical part to which both Young and Blumberg contributed, but this, again, was an interior theory not immediately applicable to boundary problems. The list of recent papers is confined to those actually referred to and is not complete.

Lilburn Tower

Alnwick, England 


\section{References}

[1] Bageminl, F.: Curvilinear cluster sets of arbitrary functions. - Proc. Nat. Acad. Sci. U.S.A. 41,1955 , pp. 379-382.

[2] Blumberg, H.: A theorem on semi-continuous functions. - Bull. Amer. Math. Soc. 24, 1918, pp. $381-383$.

[3] -》- New properties of all real functions. - Trans. Amer. Math. Soc. 24, 1923, pp. $113-128$.

[4] -"- A theorem on arbitrary functions of two variables with applications. Fund. Math. 16, 1930, pp. 17-24.

[5] - - The measurable boundaries of an arbitrary function. - Acta Math. 65, 1935, pp. $263-282$.

[6] -》- Exceptional sets. - Fund. Math. 32, 1939, pp. 3-32.

[7] -»- Arbitrary point transformations. - Duke Math. J. 11, 1944, pp. 671-685.

[8] Cartwright, M. L., and E. F. Collingwood: The radial limits of functions meromorphic in a circular disc. - Math. Z. 76, 1961, pp. 404-410.

[9] Collingwood, E. F.: Cluster sets and prime ends. - Proceedings of the International Colloquium on the Theory of Functions, Helsinki 1957, Suomalainen Tiedeakatemia, Helsinki, 1958. = Ann. Acad. Scient. Fennicæ A. I. $250 / 6,1958$.

[10] - - Cluster sets of arbitrary functions. - Proc. Nat. Acad. Sci. U.S.A. 46, 1960, pp. 1236-1242.

[11] Collingwood, E. F., and G. Piranian: Asymmetric prime ends. - Math. Ann. 144,1961 , pp. $59-63$.

[12] Dоoв, J. L.: The boundary values of analytic functions. - Trans. Amer. Math. Soc. 35, 1933, pp. 418-451.

[13] Hausdorff, F.: Mengenlehre. - 2nd ed., Göschens Lehrbücherei I. 7, Walter de Gruyter \& Co., Berlin/Leipzig, 1927.

[14] Piranian, G.: Ambiguous points of a function continuous inside a sphere. Michigan Math. J. 4, 1957, pp. 151-152.

[15] Priwalow, I. I.: Randeigenschaften analytischer Funktionen. - Hochschulbücher für Mathematik 25, Deutscher Verlag der Wissenschaften, Berlin, 1956.

[16] TANner, R. C. H.: La symétrie locale des fonctions et ensembles arbitraires. Enseignement Math. 8, 1962, pp. 192-194.

[17] Weniaminoff, V.: Sur un problème de la représentation conforme de M. Carathéodory. - Rec. Math. 31, 1922, pp. $91-93$.

[18] Weston, J. D.: Some theorems on cluster sets. - J. London Math. Soc. 33, 1958, pp. $435-441$.

[19] Young, W. H.: On the distinction of right and left at points of discontinuity. Quart. J. Pure Appl. Math. 39, 1908, pp. 67-83.

[20] - - On some applications of semi-continuous functions. - Atti del IV Congresso Internazionale dei Matematici (Roma 1908) II, Roma, 1909, pp. $49-60$. 
[21] Young, W. H.: On the discontinuities of a function of one or more real variables. Proc. London. Math. Soc. (2) 8, 1909, pp. 117-124.

[22] - - - La symétrie de structure des fonctions de variables réelles. - Bull. Sci. Math. 52, 1928, pp. 265-280.

[23] Young, W. H., and G. Ch. Young: On the internal structure of a set of points in any number of dimensions. - Proc. London Math. Soc. (2) 16, 1917, pp. $337-354$.

[24] -»- - - - On the inherently crystalline structure of a function of any number of variables. - Proc. London Math. Soc. (2) 17, 1918, pp. 1-16. 